\title{
Observation of Point Contact Visible Luminescence from Ga-Doped ZnO Layers
}

\author{
Qing Yang, ${ }^{*}$ Xiaohong Zhang, and Xiaohong Zhou \\ Faculty of Material Science and Engineering, \\ Xi'an University of Technology, \\ 5 South Jinhua Road, Xi'an 710048, China \\ Hirokazu Tatsuoka \\ Graduate School of Engineering, Shizuoka University, \\ 3-5-1 Johoku, Naka-ku, Hamamatsu 432-8561, Japan
}

Hiroko Kominami, Kazuhiko Hara, and Yoichiro Nakanishi

Research Institute of Electronics, Shizuoka University,

3-5-1 Johoku, Naka-ku, Hamamatsu 432-8011, Japan

(Received 4 February 2015; Accepted 19 March 2015; Published 25 April 2015)

\begin{abstract}
We report the point contact visible luminescence from Ga-doped $\mathrm{ZnO}$ layers. The Ga-doped ZnO layers were grown by the thermal oxidation of $\mathrm{ZnS}$ substrates with gallium in the air. The injected light emission was observed around the point-contact surface of $\mathrm{ZnO}$ layers when a forward DC voltage in the range of 2.5-9.8 $\mathrm{V}$ was applied (point contact was positive). Typically, we illustrated the luminescent spectrum for $6.7 \mathrm{~V}$ which showed a wide emission band centering at $680 \mathrm{~nm}$.
\end{abstract}

[DOI: $10.1380 /$ ejssnt.2015.201]

Keywords: Zinc oxide; Ga doping; Oxidation; Electroluminescence; Point contact; Visible emission

\section{INTRODUCTION}

It is well known that the concentration of hole-electron pairs can be increased above the equilibrium value by optical excitation or injection of minority carriers at a $p$ - $n$ junction or at a metal contact. The point contact luminescence of semiconductors, such as $\mathrm{SiC}$ [1], Ge [2] and diamond [3], has been reported previously. The nature of the contact to the semiconductor determines whether there occurs the well-known injection of minority carriers when forward voltage is applied. However, the minority carriers are generally created by minority carrier injection under strong forward-bias conditions [4]. Therefore, to obtain the injected luminescence under low voltage excitation is expected.

As a simple technology of direct conversion of electric energy into visible light, electroluminescence (EL) can be performed easily in industrial application. With a wide direct band-gap of $3.37 \mathrm{eV}$ and a large exciton binding energy of $60 \mathrm{meV}$ at room temperature, $\mathrm{ZnO}$ is recognized as one of the promising semiconductors for optoelectronic device applications [5]. To meet the challenges of light emitting diode and flat panel display, many research groups have been focusing on the electroluminescence of $\mathrm{ZnO}$ with a $p$ - $n$ junction [6-9]. However, the difficulty in synthesis of high efficient and stable p-type $\mathrm{ZnO}$ or growth of heterogeneous semiconductors with small interfacial lattice mismatch restricts the fabrication of ZnO-based LED with homogeneous or heterogeneous $p$ $n$ junctions [9-12]. Therefore, in recent years, several research groups focused on the low voltage exciting EL of $\mathrm{ZnO}$ nanowires, nanorods and microwires with metalsemiconductor (MS) junctions [13-15]. In this paper, we

* Corresponding author: yangqing@xaut.edu.cn report the point contact luminescence of Ga-doped $\mathrm{ZnO}$ layers by excitation of low voltage at room temperature.

\section{EXPERIMENTAL}

The Ga-doped $\mathrm{ZnO}$ layers were grown by the thermal oxidation of ZnS substrates with molten gallium in the air. The oxidation temperature and time are in the range of $700-900^{\circ} \mathrm{C}$ and $1-24 \mathrm{~h}$, respectively. The experimental procedure was described in detail previously [16]. The doping of $\mathrm{Ga}$ in $\mathrm{ZnO}$ was confirmed by XPS [17].

To perform the point contact luminescence of Ga-doped $\mathrm{ZnO}$ layer, an Au film with a small area was sputtered onto the ZnO layer, while a steel needle probe was directly touched to the surface of the $\mathrm{ZnO}$ layer. When a DC voltage ranging from 2.5 to $9.8 \mathrm{~V}$ (point contact is positive) was applied, the light emission could be observed around the contact point. In addition, the brightness of the emitted light increased with the current. However, when the current direction was reversed, the light emission disappeared. $N$-type of the Ga-doped $\mathrm{ZnO}$ layers was confirmed by hot-point probe measurement, which reveals the minority carrier injection under forward voltage.

\section{RESULTS AND DISCUSSION}

To evaluate the metal $/ n-\mathrm{ZnO}$ point contact, two probes touched the surface of Ga-doped $\mathrm{ZnO}$ layer, and the current-voltage characteristics were traced at room temperature by an oscilloscope. As shown in Fig. 1, the I-V curve shows a nonlinear shape, which indicates the formation of Schottky contact between the probe and $n$ - ZnO layer. Due to the small radius of probe tip, the diffusion of injected carriers at point contact is much better 


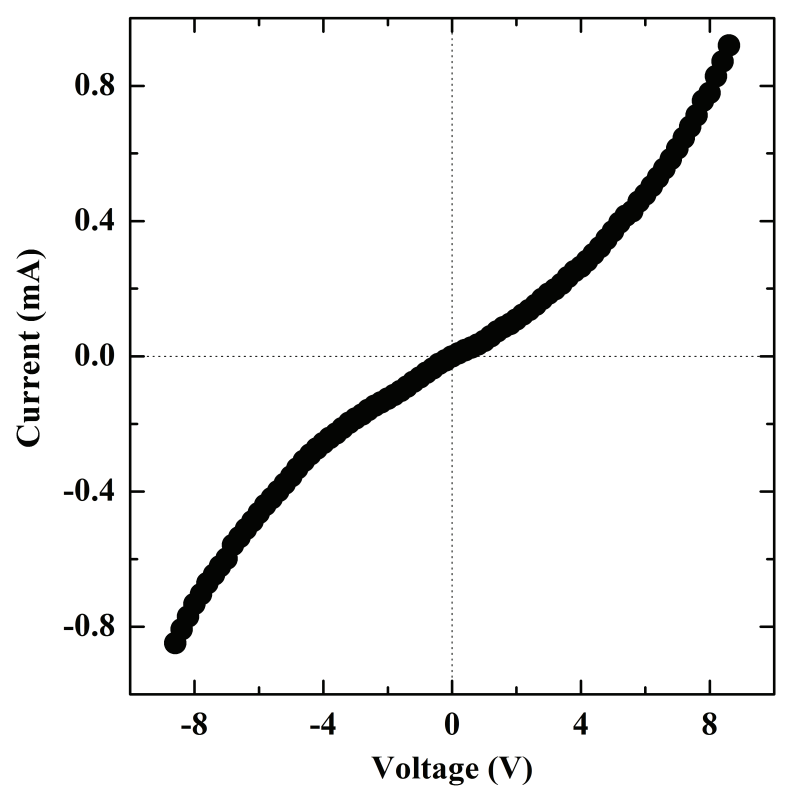

FIG. 1. Room temperature current-voltage characteristics between two probes and Ga-doped $\mathrm{ZnO}$ layer.

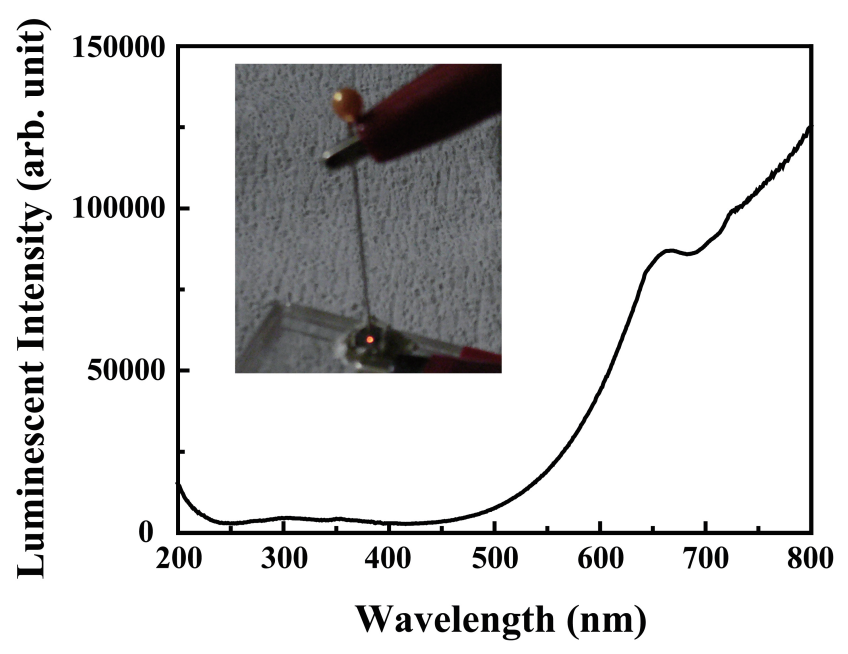

FIG. 2. Luminescent spectrum of Ga-doped ZnO layer measured at room temperature by feeding a forward direct current of $532 \mathrm{~mA}$ at $7.6 \mathrm{~V}$. The image of the corresponding emitted light was taken by a camera.

than at surface contact, which leads to the high-efficiency injection. Thus, the minority carriers (holes) are easily injected into $n$ - $\mathrm{ZnO}$ under forward voltage by tunneling through the surface potential barrier [4]. Therefore, it is considered that the light emission originating from the $\mathrm{ZnO}$ layer was due to the recombination of the injected minority carriers (holes) with the $n$-type majority carriers (electrons) at the contact point. On the other hand, it should be mentioned that no light emission was observed from undoped $\mathrm{ZnO}$ (namely grown without gallium), even if high voltage was applied. It may suggest the key role of gallium impurity in the injected light emission from $\mathrm{ZnO}$ layers.

To collect the luminescent spectrum by a spectrophotometer, a large injection was applied at $7.6 \mathrm{~V}$ and 532 $\mathrm{mA}$. As shown in Fig. 2, the spectrum shows a wide visible emission band at $680 \mathrm{~nm}$ corresponding to the observed emission light. The upward spectrum to infrared emission band indicates that heat was generated which could be owing to lattice vibration. The weak UV emission band could be ignored here. In addition, the luminescent spectrum is quite different from the photoluminescence (PL) spectra [16-18], which could be owing to their different excitation mode. For photoluminescence, electrons are excited to conduction band from valance band by short wavelength laser. In the case of the light emission, electrons are injected into the elevated energy states of the $\mathrm{ZnO}$ crystals. Meanwhile, the different excitation energy should also be responsible for the changed luminescence. The similar phenomena were also reported [19], which suggest the easy emission with long wavelength in EL spectra than in PL spectra.

\section{CONCLUSION}

In summary, the point contact visible luminescence was observed from the Ga-doped $\mathrm{ZnO}$ layers grown by the thermal oxidation of $\mathrm{ZnS}$ substrates with gallium. The forward DC voltage could range from 2.5 to $9.8 \mathrm{~V}$. Typically, the light emission observed at $6.7 \mathrm{~V}$ showed a wide emission band centering at $680 \mathrm{~nm}$.

\section{ACKNOWLEDGMENTS}

This work was supported by National Natural Science Foundation of China (No. 51202191), Natural Science Basic Research Plan in Shaanxi Province of China (No. 2012JQ6002), and Scientific Research Program Funded by Shaanxi Provincial Education Department of China (No. 12JK0427).
[1] O. V. Losev, Phil. Mag. 6, 1024 (1928).

[2] W. H. Brattain and J. Bardeen, Phys. Rev. 74, 231 (1948).

[3] R. Wolfe and J. Woods, Phys. Rev. 105, 921 (1957).

[4] E. F. Schubert, Light-emitting diodes, 2nd ed. (Cambridge University Press, 2006).

[5] D. Panda and T. Y. Tseng, J. Mater. Sci. 48, 6849 (2013).

[6] S. J. Jiao, Z. Z. Zhang, Y. M. Lu, D. Z. Shen, B. Yao, J. Y. Zhang, B. H. Li, D. X. Zhao, X. W. Fan, and Z. K.
Tang, Appl. Phys. Lett. 88, 031911 (2006).

[7] P. Chen, X. Ma, and D. Yang, Appl. Phys. Lett. 89, 111112 (2006).

[8] T. Ohashi, K. Yamamoto, A. Nakamura, and J. Temmyo, Jpn. J. Appl. Phys. 47, 2961 (2008).

[9] Y. S. Choi, J. W. Kang, D. K. Hwang, and S. J. Park, IEEE Trans. Electron Dev. 57, 26 (2010).

[10] A. Janotti and C. G. Van de Walle, Rep. Prog. Phys. 72, 126501 (2009). 
[11] C. H. Park, S. B. Zhang, and S. H. Wei, Phys. Rev. B 66, 073202 (2002).

[12] Ya. I. Alivov, E. V. Kalinina, A. E. Cherenkov, D. C. Look, B. M. Ataev, A. K. Omaev, M. V. Chukichev, and D. M. Bagnall, Appl. Phys. Lett. 83, 4719 (2003).

[13] Y. X. Wang, Q. F. Zhang, H. Sun, Y. L. Chang, and J. L. Wu, Acta Phys. Sin. 57, 1141 (2008).

[14] N. Bano, I. Hussain, O. Nur, M. Willander, H. S. Kwack, and D. Le Si Dang, Appl. Phys. A 100, 467 (2010).

[15] M. Ding, D. Zhao, B. Yao, B. Li, Z. Zhang, C. Shan, and D. Shen, J. Phys. D: Appl. Phys. 44, 075104 (2011).
[16] Q. Yang, Y. Saeki, S. Izumi, T. Nukui, A. Tackeuchi, A. Ishida, and H. Tatsuoka, Appl. Surf. Sci. 256, 6928 (2010).

[17] X. H. Zhou, Q. Yang, J. T. Zou, and S. H. Liang, Acta Phys. Sin. 64, 087803 (2015).

[18] Q. Yang, X. Zhou, T. Nukui, Y. Saeki, S. Izumi, A. Tackeuchi, H. Tatsuoka, and S. Liang, Sci. China Tech. Sci. 57, 2500 (2014).

[19] L. Yi, Z. Xu, Y. Hou, X. Zhang, Y. Wang, and X. Xu, Chinese Sci. Bull. 46, 1223 (2001). 